\title{
Habitual protein intake appears to modulate postprandial muscle protein synthesis responses to feeding in youth but not in older age
}

Protein ingestion plays a critical role in muscle mass maintenance and growth, as a key regulator of skeletal muscle protein synthesis (MPS) ${ }^{(1)}$. Sarcopenia, defined as an age-related loss of muscle mass and function, is a key contributing factor to frailty, a syndrome with prominent impact on maintenance of physical independence ${ }^{(2)}$. Anabolic resistance, the blunted MPS response of skeletal muscle to key anabolic stimuli, namely essential amino acid (EAA) nutrition ${ }^{(3)}$, has been demonstrated in older individuals. Therefore, it is likely that anabolic resistance contributes to sarcopenia progression.

Despite a wealth of evidence demonstrating the role of protein nutrition for MPS stimulation, limited research has investigated the effect of habitual protein intake on MPS across distinct phases of adulthood (i.e. youth versus older age).

This study investigated the effect of habitual protein intake in young $(\mathrm{n}=11,25.3(3.5 \mathrm{y}))$ and older $($ non-sarcopenic, $\mathrm{n}=16,70.2$ (3.2y)) community-dwelling adults (63\% male) on myofibrillar MPS responses to feeding. Protein intake was determined using 4-day diet diaries analysed using Microdiet software (v5, Downlee Systems Ltd, UK). Post-absorptive MPS and MPS in response to mixed macronutrient feeding ( $4.25 \mathrm{x}$ basal metabolic rate; $16 \%$ protein) was determined from muscle biopsies of the $m$. vastus lateralis (VL) via stable isotope tracer $\left(\left[1,2-{ }^{13} \mathrm{C} 2\right]\right.$-leucine $)$ infusions paired with mass-spectrometric analyses. VL protein expression of anabolic signalling targets was determined via immunoblotting. Unpaired t-tests were used to determine differences in habitual dietary intake between groups, with linear regression analysis used to investigate the relationship between components of habitual diet with MPS and anabolic signalling.

Habitual protein intake (g.kg-1.day-1) was not different between the age-groups (Old: 1.28 (0.34), Young: 1.13 (0.23) g.kg- 1.day-1, $P=0.19$ ) and was not associated with post-absorptive MPS in young or older adults (Young: R2 $=0.01, P=0.80$, Old: R2 $=0.0004$, $P=0.96)$. Conversely, in young individuals, higher habitual protein intake was associated with reduced fed- state MPS $(\mathrm{R} 2=0.37$, $P=0.04)$, a relationship that was not apparent in older adults $(\mathrm{R} 2=0.32, P=0.50)$. Habitual leucine intake (mg.kg-1.day-1) was significantly higher in young individuals $(P=0.05)$ but was not associated with post-absorptive or fed-state MPS in either age-group (Young, post-absorptive: R2 $=0.02, P=0.66$, Fed: R2 =0.26, $P=0.12$; Old, post-absorptive: R2 $=0.14, P=0.96$, Fed: R2 $=0.0003$, $P=0.96)$. No significant associations were observed between any anabolic signalling target and habitual dietary protein values in either age-group.

The findings of this study identify an interesting difference between the protein intake habits of healthy young and older individuals showing lower leucine, but not total protein intake in older age. In addition, in younger individuals only, higher habitual protein intake appears to negatively impact acute muscle protein metabolic responses to a key anabolic stimulus (i.e. protein nutrition). Further research is needed to fully determine the relationship between habitual protein intake and muscle protein metabolic responses across the life-course.

\section{References}

1. Atherton PJ \& K Smith (2012) J Physiol 590, 1049-1057.

2. Cruz-Jentoft A, Baeyens JP \& Bauer JM et al.. (2010) Age Ageing 39, 412-423.

3. Cuthbertson D, Smith K \& Babraj J et al.. (2005) FASEB J 19, 422-424. 